Research Article

\title{
Research on Modern Book Packaging Design under Aesthetic Evaluation Based on Deep Learning Model
}

\author{
Yuanyuan Li (iD \\ Graduate School of Art, Chung-Ang University, Seoul 06911, Republic of Korea \\ Correspondence should be addressed to Yuanyuan Li; liyuanyuan840101@st.btbu.edu.cn
}

Received 12 October 2021; Revised 25 October 2021; Accepted 26 October 2021; Published 8 November 2021

Academic Editor: Gengxin Sun

Copyright (C) 2021 Yuanyuan Li. This is an open access article distributed under the Creative Commons Attribution License, which permits unrestricted use, distribution, and reproduction in any medium, provided the original work is properly cited.

\begin{abstract}
The development of information technology has led to the rapid development of modern book packaging design. Book packaging design is different from painting. It is a kind of design process that combines artistry and practicality and has double characteristics. With the continuous progress of society, people's requirements for book packaging design have become higher and higher, and modern book packaging design has become an important topic in the field of art design. To this end, this paper introduces the machine learning algorithms used in this paper, including the AdaBoost algorithm and the SVR algorithm. Specifically, it includes the principles and specific implementation steps for AdaBoost classification algorithm and SVR regression algorithm, as well as evaluation indexes of AdaBoost classification and SVR regression analysis. Realization of physical books reflects artistry, creativity, professionalism, popularity, and vitality of books in the packaging and design of books in the electronic information era. The stimulation effect of this paper starts from packaging design, inspection mechanism, and brand psychology to get the superior design in modern book packaging design.
\end{abstract}

\section{Introduction}

With the advent of the electronic information era, virtual electronic books have rapidly occupied the market with a strong momentum and gained the favor of the majority of consumers, especially young people $[1,2]$. Electronic books have a serious impact on the whole traditional publishing industry, making it face a serious crisis of survival. Book packaging design occupies an important position in the traditional publishing industry and has a nonnegligible impact on the sales of books. Therefore, the influence of electronic devices on it is also very big [3, 4]. How to follow the trend of the time in the information age, cater to the needs of consumers, and establish a sustainable development mechanism for modern book packaging design is a common problem faced by every traditional publishing enterprise.

Book packaging design refers to the overall design of a book or a series of books by combining the sales method and readership of the book with the theme design for marketing purposes, as well as the design of the cover and jacket and the establishment of the corresponding brand image [5]. For book designers, in order to break the impact of new electronic books on traditional books and make paper books occupy the market share, they must reflect the spatial difference between the packaging design of books and electronic books, fully express the aesthetic and cultural atmosphere lacking in electronic books in book design, and pass them to readers, so that readers are psychologically more willing to use paper books. It can be seen that modern book packaging design is actually a study that combines language, text, painting, aesthetics, and art into one, which can reflect the connotation and aesthetic interest of the designer and, at the same time, play an effective role in improving the readers' aesthetic ability, their own temperament, and life interest [6].

The art and culture level shown in the modern book design can show the cultural heritage of the country or nation to a certain extent. In the packaging design of modern books, how to reflect both the traditional national culture and contain modern fashion style is more closely related to the design level and design standards in book design [7]. With a history of five thousand years, the Chinese nation has 
a deep cultural heritage, a long history, and deep roots in the artistic tradition, so there are a lot of materials that can be used in the packaging design of books. In modern book packaging design, the traditional Chinese culture should be combined with modern design concepts and ideas, design methods, and advanced high-tech means with a vision of development and advancement, unifying national tradition and modernity, so as to design Books that can withstand the impact of time $[8,9]$.

In the initial stage of human civilization, there was an extreme lack of writing materials, because characters were inscribed on materials such as animal bones and oracle bones, so generally the whole picture was almost distributed with words, and there was little space for making designs [10]. With the development of productivity, paper making and printing appeared one after another, and people's writing materials increased rapidly, which greatly promoted the development of people's design consciousness and made China's design level ahead of other countries in the world. In the Tang and Song dynasties, the packaging of books, the design of New Year paintings, and other printed materials used woodblock printing in printing. For example, the illustrations of the Ming and Qing dynasties, such as the Four Beautiful Beauties and the Diamond Sutra, and some folk of New Year paintings with various shapes and bright colors, pay attention to the decorative role and flatness of the picture, do not pay much attention to the depth of perspective, and are influenced by the composition of paintings in the arrangement of the text, and some combine color blocks and frame lines to show their uniqueness, all of which show the people's design consciousness and high aesthetic ability [11].

For a long time, aesthetics has been subordinated to the research in the field of philosophy. With the development of computer technology and signal processing technology, researchers in various countries have begun to combine aesthetic research with computer technology to explore computable methods of aesthetics. Aesthetic standards are relatively fixed scales used to measure and evaluate the aesthetic value of objects. In a narrow sense, aesthetics is a comprehensive product of composition, color, light, and expression of mood and is a discipline that shows natural beauty through painting, color, layout, etc. The purpose of aesthetics is to obtain a sense of natural beauty $[12,13]$. The use of mathematical models to quantitatively describe aesthetic criteria dates back to 1933 . And with the development of machine learning, it is possible to design book covers using machine learning. For this purpose, this paper introduces the machine learning algorithms used in this paper, including the AdaBoost algorithm and SVR algorithm [14]. Specifically, it includes the principles and specific implementation steps for AdaBoost classification algorithm and SVR regression algorithm, as well as evaluation indexes of AdaBoost classification and SVR regression analysis. Realization of physical books reflects artistry, creativity, professionalism, popularity, and the vitality of books in the packaging and design of books in the electronic information era. In this paper, the simulation effect starts from packaging design, inspection mechanism, and brand psychology to get the superior design in modern book packaging design.

\section{Related Work}

From the above research results, we can see that book cover complexity is an important metric for measuring the aesthetic value of book covers, and it is of great significance for assessing the aesthetic value of book covers. However, there is a lack of effective experimental validation and application of aesthetics-related book cover complexity research [15].

At the same time, in the fields of painting, sculpture, multimedia film, and television, there is a long-standing and profound understanding of the construction of beauty, such as symmetry, balance, combination, direction, color harmony, and color contrast [16]. In addition, when discussing the aesthetics of book covers from the perspective of photography, a perfect image must have a clear focus, correct exposure, and a flawless appearance.

There are three general criteria: (1) clear expression of the photographic object, the subject; (2) focus on that object; and (3) simplification achieved by eliminating distracting elements. For color photographs, more attention is usually paid to color fidelity, color saturation, color balance, color contrast, etc. [17]. All these can provide useful ideas for the aesthetic calculation of book covers. However, these expressions and descriptions exist only in the artistic category, and how to quantify and calculate them is a difficult problem, and scholars from various countries have explored and studied the theories, computable models, and applications related to book cover aesthetics from different perspectives.

Automatically assessing the aesthetic value of book covers is the focus of research on computable book cover aesthetics for a wide range of objects, such as photographic photographs, paintings, computer graphics, diagrams, canvases, and composites [18]. Among the studies on book cover aesthetics, a research group led by [19] was one of the first to achieve the quantification of book cover features on the aesthetic quality of book covers, mainly by processing and analyzing user uploaded book cover data in the online book cover system photo. [20] to achieve the correlation from visual features of book covers to the aesthetic determination of book covers, which defined and extracted 35 book cover features including brightness, color distribution, wavelet transform, region composition, and depth of field, based on some simple rules used in photography and used feature selection to get the most effective 15 features to classify book covers with high and low aesthetics using Support Vector Machine (SVM). The correct classification rate of $70.12 \%$ was obtained, and then multiple linear regression was used to predict the book cover beauty value using book cover features [21]. Although the prediction result is not high in correctness, it shows that it is still feasible to predict book cover beauty score using book cover features. Based on this research, the Aesthetics Quality Inference Engine (ACQUINE) aesthetic quality reference system [22] was also developed. ACQUINE is a typical online book cover aesthetics score and search engine; the project started in 2005 and went online in April 2009; currently, users can upload their photos and automatically obtain the aesthetic score of the photos. The system is mainly used to assess the aesthetic quality of professional color photographs. 
Shen et al. [23] refer to [24] for predicting the aesthetic description of book covers: "good," "bad," or "ugly." However, they did not propose a method to improve the correct rate of classification prediction. In addition, Liu et al. [25] extracted visual features of book covers to identify highquality professional photos and low-quality snapshots. While Hammad and El-Sankary [26] proposed to classify professional photos and snapshots by visual saliency region enhancement and used visual perception (including sharpness, exposure, and texture) to build a visual saliency region model to achieve better aesthetic classification of book covers than global features, Geyer [27] analyzed the technical characteristics related to professional photography photos and used Blur Sense Detection to roughly estimate the focused subject area and extract features from the separated subject area, which effectively improved the classification. In addition, Zhou et al. [28] analyzed feature selection from the perspective of artistic aesthetics for paintings, extracted global features such as color distribution, luminance, haze effect, and edge distribution, combined them with local features after book cover segmentation, and used subjective visual survey results for machine learning using the AdaBoost algorithm to achieve aesthetic visual quality category classification. Wei et al. [29] analyzed the validity of self-similarity of aesthetic book covers based on Pyramid Histogram of Oriented Gradients (PHOG) features and wanted to use it in the subsequent classification of high and low aesthetics of book covers.

The results of the aesthetic value assessment of book covers can also be applied in book cover retrieval. For example, Li [30] explored the book cover aesthetic analysis by introducing a book cover aesthetic algorithm in a book cover query system, where the aesthetic score is calculated from a combination of measurements such as regional sharpness, contrast, and color concentration of the most aesthetically attractive relevant areas after the book cover segmentation. Book cover retrieval system that introduces this algorithm can filter book covers that better meets user needs.

Generally speaking, the study of book cover aesthetics has only emerged internationally in recent years and has gradually gained the attention of relevant researchers. The research abroad is still in its infancy, and we have not yet seen any relevant research results on book cover aesthetics in China. Due to the subjectivity and complexity of aesthetics, the study of book cover aesthetics is still in the preliminary exploration and embryonic stage, with a large number of issues to be studied and no mature methodologies so far.

\section{Book Cover Beauty Level Classification Algorithm}

3.1. Selection of Classification Algorithms. For the process of classifying book cover aesthetic rating, which is a mapping process established between book cover features and book cover aesthetic rating, it can be implemented by classification algorithms. Classification algorithms include unsupervised classification and supervised classification algorithms. The unsupervised classification method is a method to classify samples into categories based on their distribution without a priori knowledge (book cover aesthetic information) [24,31], while supervised algorithm is a statistical method with high classification accuracy; based on the premise of known category labels, effective features are extracted for each category of training samples, and the samples are classified into each category label by discriminant function or rule. Since this paper is based on the psychology book cover aesthetics data of a large audience to achieve book cover aesthetics class classification, the supervised classification method is used. In recent years, the mainstream supervised classification methods are SVM and AdaBoost algorithms, and the SVM algorithm has shown greater advantages for small sample data, nonlinear and high-dimensional pattern recognition problems. Compared with other machine learning algorithms, the AdaBoost algorithm is simple to implement, has the characteristics of stable decrease in the upper bound of classification error rate with the increase of training times, does not appear overfitting, and has excellent classification ability for large sample data [31, 32]. Therefore, considering the large amount of data used in this paper and the relatively high feature dimension of each sample, the AdaBoost algorithm is used for the classification of book covers in terms of aesthetic rank. Meanwhile, the experimental results in this experiment also verify that AdaBoost has better classification effect compared with SVM.

Therefore, this paper uses the AdaBoost algorithm to build a book cover aesthetic rating evaluation model by training and learning the book cover aesthetic rating data to predict the aesthetic rating of book covers.

\section{Introduction of AdaBoost Classification Algorithm}

AdaBoost algorithm was first proposed in [33] as an adaptive iterative algorithm based on Boosting, which has a higher efficiency than Boosting. Currently, the AdaBoost algorithm belongs to feature classification algorithm, which is extremely important in machine learning, and it is widely used in facial expression recognition, book cover retrieval.

The core idea of the AdaBoost algorithm is to train different weak classifiers on the same training set and then aggregate these weak classifiers to construct a strong classifier. In essence, it is a boosting process for a simple weak classification, which gradually improves the data classification ability during the continuous training and learning process. During the training process, the AdaBoost classifier can exclude feature data with little relevance and has a feature selection function. The AdaBoost algorithm has the following advantages: (1) it has high accuracy; (2) it provides a basic framework to construct subclassifications by various methods; (3) it is suitable for training and learning on large sample data; (4) the classifier itself has a feature selection function. No feature screening is required before training.

4.1. Implementation of AdaBoost Classification Algorithm. The AdaBoost algorithm is implemented by changing the distribution of sample data. In each training set, we first 
check whether each sample is correctly classified or not and then modify the weight of each sample in combination with the previous overall classification accuracy [34]. Then, the new data with modified weights are sent to the lower classifier and trained, and finally the weak classifiers obtained from each training are fused together as the final decision classifier.

Since this paper performs the classification of book covers with high and low aesthetics in two categories, the basic AdaBoost two-class classification algorithm is used to learn the feature data and obtain the optimal classifier. The details are described as follows:

(1) For a given training sample $\left(\vec{x}_{1}, y_{1}\right),\left(\vec{x}_{2}, y_{2}\right), \ldots,\left(\vec{x}_{n}, y_{n}\right)$, where $\vec{x}_{i}$ denotes the feature vector of samples in the feature data, each value in the vector corresponds to each feature value of the book cover sample. Assuming that each sample extracts a K-dimensional feature vector, then $\vec{x}_{i}$ has $\mathrm{K}$ dimensions. $\mathrm{N}$ is the total number of training samples, and for two-class classification, $y_{i}=-1$ denotes negative samples and $y_{i}=1$ denotes positive samples.

(2) Next, the weights of each sample are initialized $D_{1}(i)=1 / n, i=1, \ldots, n$.

(3) For $t=1, \ldots, T \partial$ (where $T$ is the number of training rounds)

(1) For each feature, by training a weak classifier, and calculating the weighted $w_{t}$ error rate $\varepsilon_{t, k}$ of the results obtained by classifying all samples by this weak classifier (where $k$ corresponds to a specific one of the K-dimensional features, $1 \leq k \leq K$ ).

$$
\varepsilon_{t, k}=\sum_{i=1}^{n} D_{t}(i)\left[y_{i} \neq h_{t, k}\left(x_{i}\right)\right] .
$$

(2) Select the optimal weak classifier, $h_{t}$ (because each $h_{t, k}$ corresponds to a weighted error rate $\varepsilon_{t, k}$, so $h_{t}$ is the weak classifier corresponding to the smallest $\left.\varepsilon_{t, k}\right)$.

(3) Calculate the weights of this optimal weak classifier in the final synthesis of the strong classifier:

$$
\alpha_{t}=\frac{1}{2} \ln \left(\frac{1-\varepsilon_{t}}{\varepsilon_{t}}\right)>0 .
$$

(4) Based on the optimal weak classifier $h_{t}$ for that round, for each sample, the weights are readjusted [5]

$$
D_{t+1}(i)=D_{t}(i)\left(\sqrt{\frac{\varepsilon_{t}}{1-\varepsilon_{t}}}\right)^{y_{i} h_{h}\left(x_{i}\right)} \text {. }
$$

The normalization of this equation is shown as follows:

$$
D_{t+1}(i)=\frac{D_{t+1}(i)}{\sum_{i=1}^{n} D_{t+1}(i)} .
$$

(4) Obtain the final strong classifier as

$$
H_{f \text { final }}(x)=\operatorname{sign}\left(\sum_{t=1}^{T} \alpha_{t} h_{t}(x)\right) \text {, }
$$

where the value of weak classifier $h_{k}\left(x_{i}\right)$ is 1 or -1 .

First, after the first weak classifier (classification function of $\left.h_{i}\left(x_{i}\right)\right)$, the error of $\varepsilon_{1}=0.3$, $\alpha_{1}=0.5 \times \ln (1-\varepsilon / \varepsilon)=0.5 \times \ln (0.7 / 0.3)=0.42$. Then, according to the weight update and weight normalization process in AdaBoost algorithm description $C$, the weights of the positive samples that are misclassified are 1/6 (3), and the weights of all other samples are 1/14 (7); after the second classifier (classification function $h_{2}\left(x_{i}\right)$ ), the error of the weak classifier $\varepsilon_{2}=0.21$, weight $\alpha_{2}=0.65$. The same weight update and weight normalization process is performed; after the third classifier (classification function $h_{3}\left(x_{i}\right)$ ), the error $\varepsilon_{3}=0.14$ and the voting weight $\alpha_{3}=0.92$. The strong classifier form by the three classifier process is

$$
H(x)=\operatorname{sign}\left(0.42 \times h_{1}\left(x_{i}\right)+0.65 \times h_{2}\left(x_{i}\right)+0.92 \times h_{3}\left(x_{i}\right)\right) .
$$

Therefore, we can find the values of regions $\mathrm{A}, \mathrm{B}, \mathrm{C}, \mathrm{D}, \mathrm{E}$, and $\mathrm{F}$ as

$$
\begin{aligned}
& A: H(x)=\operatorname{sign}(0.42 \times 1+0.65 \times 1+0.92 \times 1)=\operatorname{sign}(1.99)=1, \\
& B: H(x)=\operatorname{sign}(0.42 \times(-1)+0.65 \times 1+0.92 \times 1)=\operatorname{sign}(1.05)=1, \\
& C: H(x)=\operatorname{sign}(0.42 \times(-1)+0.65 \times(-1)+0.92 \times 1)=\operatorname{sign}(-0.15)=-1, \\
& D: H(x)=\operatorname{sign}(0.42 \times 1+0.65 \times 1+0.92 \times(-1)=\operatorname{sign}(0.15)=1, \\
& E: H(x)=\operatorname{sign}(0.42 \times(-1)+0.65 \times 1+0.92 \times(-1)=\operatorname{sign}(-0.69)=-1, \\
& F: H(x)=\operatorname{sign}(0.42 \times(-1)+0.65 \times(-1)+0.92 \times(-1))=\operatorname{sign}(-1.99)=-1 .
\end{aligned}
$$

So, how to detect $\mathrm{A}, \mathrm{B}$, and $\mathrm{D}$ (light gray area) is judged as a positive sample area, while $C, E$, and $\mathrm{F}$ (white area) are judged as a negative sample.
The above analysis shows that, in the above example, with only ten samples, a strong classifier composed of three weak classifiers can obtain $100 \%$ classification accuracy. 


\section{Book Cover Aesthetics Score Evaluation Model}

5.1. Introduction to SVR Algorithm. SVR is an application of support vectors to the function regression problem and is another application of the SVM algorithm. So, before introducing the SVR algorithm, let us first to understand the SVM algorithm. The SVM algorithm adopts the kernel function mapping principle and also introduces the concept of structural risk. Compared with traditional methods, it solves the problem of large sample data, facilitates the treatment of nonlinear problems, solves the problems of local minima and dimensional catastrophes, and is therefore widely used.

The basic idea of SVM is to map a low-dimensional data space to a high-dimensional space (Hilbert space) using a nonlinear mapping, which essentially transforms the input low-dimensional data space to a high-dimensional space with the help of a linear transformation (defined by the inner product function) and then finds the optimal hyperplane in this high-dimensional space [13].

The SVM algorithm is proposed for the classification problem and is described mathematically as follows, for a given training set:

$$
T=\left\{\left(x_{1}, y_{1}\right), \ldots,\left(x_{l}, y_{l}\right) \mid x_{j} \in R^{n}, y_{j} \in R\right\},
$$

where $R^{n}$ is the input space, where each point $x_{i}$ consists of $n$ attributes and $y_{j} \in R=\{1,2, \ldots, m\}, j=1, \ldots, l, m$ is the number of $x_{i}$ possible corresponding categories. By finding a real-valued function $g(x)$ on $R^{n}$, it is possible to use the decision function $f(x)=\operatorname{sng}(g(x))$ to infer the y-value problem corresponding to any one $x$. For the classification problem, the points on $R^{n}$ are essentially divided into $m$ subspaces. For the regression problem, the above mathematical model also applies, and the inferred y-values correspond to continuous values; i.e., the points on 55 are divided into the corresponding mapping functions. Vapnik, in order to apply support vectors to the functional regression problem, introduces an insensitive loss function, defined as

$$
\begin{aligned}
e(y, f(x, a)) & =e\left(|y-f(x)|_{c}\right), \\
|| y-\left.f(x)\right|_{c} & = \begin{cases}0 & |y-f(x)| \leq \varepsilon, \\
|y-f(x)|_{c} .\end{cases}
\end{aligned}
$$

This loss function indicates a loss of 0 if the error of the actual value with respect to the predicted value is less than $e$. In this process, the approximation of the function is solved by introducing an insensitive loss function.

\section{Aesthetic Score Prediction Evaluation Index}

In this paper, we consider various evaluation indexes and use correlation coefficient, root mean square error, mean absolute error, the mean relative error, residual standard deviation, and coefficient of determination of the regression model to measure the performance of SVR regression.

First, $X$ is the actual value of the sample in the regression prediction, $\mathrm{Y}$ is the predicted target value, $n$ represents the number of samples, $i=1,2, \ldots, \mathrm{n}, \bar{X}$ is the average of the actual value of all samples, and $\bar{Y}$ is the average of the predicted value of all samples [16].

(1) Correlation coefficient refers to the deviation of the predicted target value from the sample target value in the regression prediction. The range of correlation coefficient is $0-1$, where 1 means very correlated, and 0 means not correlated. The correlation coefficient is significant when $0.5<R<0.8$ and highly correlated when $0.8<R<1$. The calculation formula is shown as follows:

$$
R=\frac{\sum_{i=1}^{n}\left(X_{i}-\bar{X}\right)\left(Y_{i}-\bar{Y}\right)}{\sqrt{\sum_{i=1}^{n}\left(X_{i}-\bar{X}\right)^{2} \sum_{i=1}^{n}\left(Y_{i}-\bar{Y}\right)^{2}}} .
$$

(2) Root mean square error (RMSE), which indicates the degree of dispersion of the sample, is calculated as

$$
\mathrm{RMSE}=\sqrt{\frac{\sum_{i=1}^{n}\left(X_{i}-Y_{i}\right)^{2} / Y_{i}}{n} .}
$$

(3) Mean absolute deviation (MAD):

$$
\text { MAD }=\frac{\sum_{i=1}^{n}\left|X_{i}-Y_{i}\right|}{n} .
$$

(4) Mean absolute relative deviation (MARD):

$$
\text { MARD }=\frac{\sum_{i=1}^{n}\left|\left(X_{i}-Y_{i}\right)\right| / Y_{i}}{n} .
$$

(5) The residual standard deviation S, also known as the mean squared deviation, is the value of the arithmetic square root of the mean square of the error. Its magnitude reflects the accuracy of the model's estimated prediction. The smaller the residual standard deviation, the higher the accuracy of the model.

$$
S=\sqrt{\frac{\sum_{i=1}^{n}\left(X_{i}-Y_{i}\right)^{2}}{n-2}},
$$

(6) The coefficient of determination of the regression model $R^{2}$, which refers to the proportion of the sum of squares of the regression to the sum of squares of the total deviations, is used to measure the goodness of fit of the estimated model to the sample observations. the closer the $R^{2}$ to 1 , the better the fit. The formula is shown as follows:

$$
R^{2}=1-\frac{\sum_{i=1}^{n}\left(X_{i}-Y_{i}\right)^{2}}{\sum_{i=1}^{n}\left(X_{i}-\bar{X}\right)^{2}} .
$$

\section{Simulation Effect}

7.1. Design Effect. Innovation is necessary in any design, so that the design can show its own characteristics and attract the eyes of consumers. There are various sources of materials for creation in modern book design, including things that 
exist in real life, as well as some myths, fables, and legends, and new things that keep appearing with the development of the time. No matter the kind of the material, it can play a certain role in the inspiration of book packaging design.

With the rapid development of computer technology in today's era, graphic design software allows designers to allocate more time to some details of design [19]. And it achieves many results that cannot be achieved by traditional design methods, which greatly broadens the available space for design. But the technology is always just a rigid technology, and to have high design works, the most important thing is always the human creativity.

As shown in Figure 1, in book design, the factors that can give readers the most intuitive visual perception mainly include fonts, colors, and graphics. The three are complementary to each other, so they should be combined in the design and strive to achieve the harmony of text, color, and graphics.

The main function of the text in Figure 1 is for the reader to read, so the arrangement should focus on easy readability and the integration of text and other elements. Specifically, firstly, the text arrangement should be accurate and clear, and the expressed information should be presented completely; secondly, on the basis of ensuring the reading effect, the text should be designed artistically, so that the arrangement of the text is both pleasing to the eye and satisfying the reading needs of the readers; finally, gradient, repetition, tilt, and other techniques can also be used for text arrangement, so that the text can show more beauty.

Different colors in Figure 1 will show different styles; for example, bright colors will generally look more gorgeous, while gray colors are relatively plain. Different colors will bring different psychological feelings to people. Therefore, the psychological feeling brought by such colors can also be used in book design, so that the appearance of the book design is consistent with the main tone that the author wants to express.

Graphic design of Figure 1 plays an important role in the design of books. The success of book design has a great deal to do with graphics and their quality. By graphics in this context, we mean graphics that are visually recognizable and have variations in texture, color, size, and shape on a flat surface. Graphic design is very important in both graphic design and color design. In graphic design, color and texture are the most fundamental and essential parts. The focus of the graphic design process is the processing and analysis of texture contrast, which is the most important visual treatment in graphic design.

\section{Simulation Performance}

A comparison of HMO-NSGA-II (our method) and NSGAII for solving the MK01 problem is shown in Figure 2.

From Figure 2, it can be seen that HMO-NSGA-II can find the optimal solution stably and quickly with good convergence; HMO-NSGA-II finally obtains 13 nondominated solutions, and NSGA-II finally obtains 8 nondominated solutions; the number of nondominated solutions obtained by HMO-NSGAII is large and widely and uniformly distributed, which reflects better global and local search performance.

ImageDataGenerator is used to expand the input data to 2000 images by randomly panning and inverting the original image and label file. Then, 15 epochs are used for training to obtain the results. According to the comparison between the training and validation sets, the initial learning rate was modified to $0.01,0.001,0.0001$, and 0.00001 , and the loss function converged best when the learning rate was 0.0001 , with a loss value of 0.0213 and an Acc metric of 0.9626 . The loss function converged well. The loss curve is shown in Figure 3.

After training, the Dice metric can reach 0.9999. The Dice coefficient plot is shown in Figure 4.

The effect of the size of the training set on the accuracy of the model was further analyzed. When the learning rate is 0.0001 , the neural network model is trained with 500, 1000, 1500,2000 , and 2500 sets of data, and 15 epochs are performed for each set. The accuracy of the model gradually improves, and the value of the loss function changes less after the number of epochs exceeds 2000, thus indicating that the model can already obtain high accuracy with 2000 sets of training data.

The design results of any commodity must be objectively tested, and books are no exception. In order for a specific book design to be approved, it must be inspected by multiple parties. In the inspection process, due to the different psychology of each inspector, inspection perspective, etc., with the focus being different, there will be a variety of differences in the inspection process, ultimately even leading to contradictory or opposing test results.

To sum up, the rapid development of electronic information technology has led to a huge impact of virtual electronic books on traditional paper books, which has caused book packaging design to be affected in no small way. Therefore, it is necessary to find out the spatial differences between electronic books and paper books and to focus on mining these differences to design book covers that are in line with the public aesthetics and the trend of the times, so as to promote the long-term development of traditional paper books and the traditional publishing industry.

\section{Discussion}

Book design is constantly changing with the times. In order to be favored by the audience, books must be combined with the trend of the time, meet the need of the public aesthetics and other aspects, and present diverse characteristics. However, at present, the design of some books in China still shows a strong implication of traditional design habits and expressions. Specifically, in the packaging design of books, the following ideas should be considered to improve the quality of book packaging design.

The manuscript is the basis for book design. In order to deeply express the content of the book manuscript in book design, it is absolutely not enough just to understand the content of the book manuscript in general, and the designer must not only understand the content of the book manuscript in detail, but also understand the details of the book 

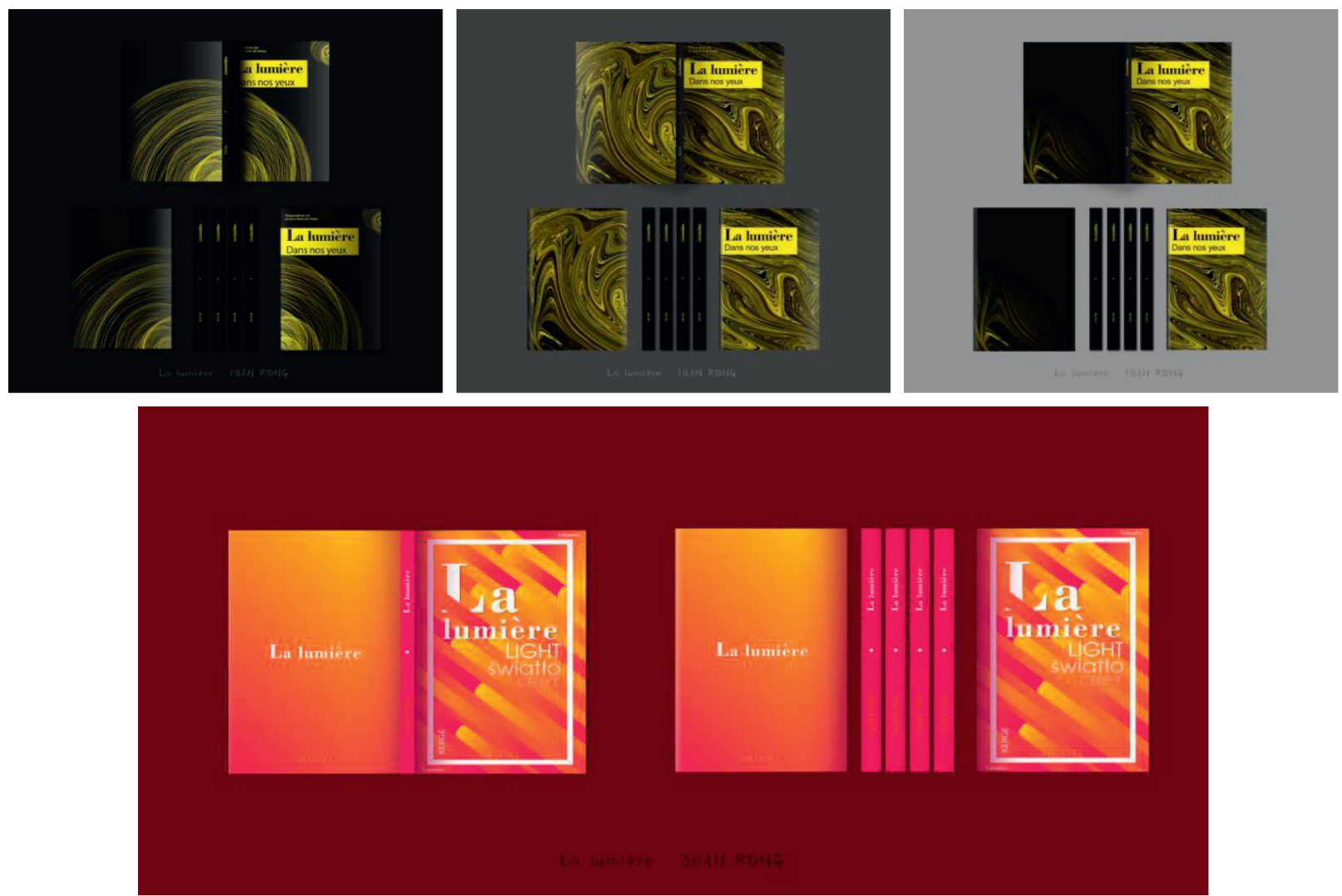

Figure 1: Different design options for book covers.

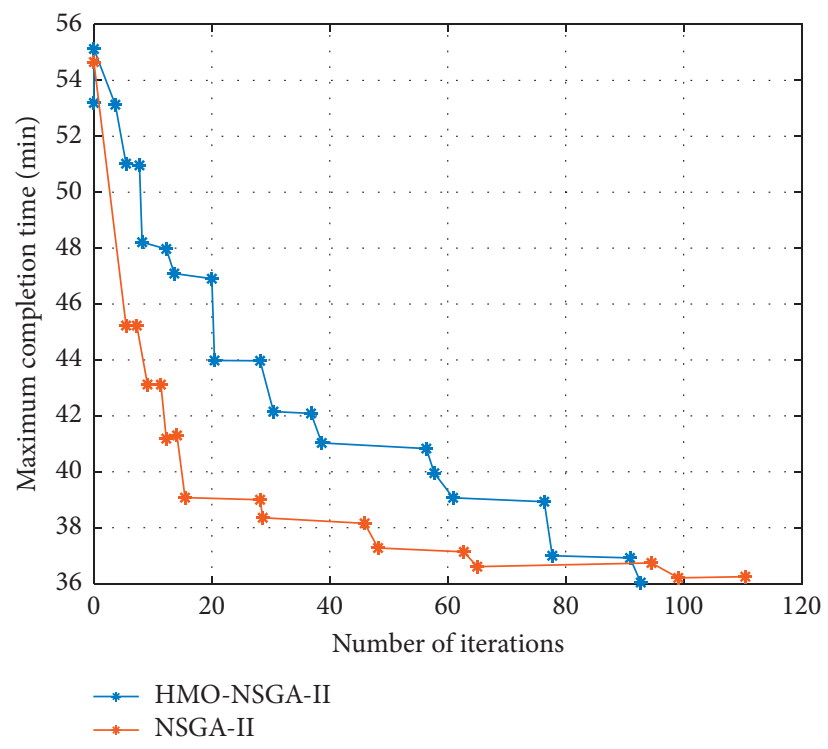

FIgURE 2: Comparison between HMO-NSGA-II and NSGA-II for solving MK01 problem.

manuscript. Specifically, the designer can first quickly browse the entire manuscript as a whole to get a preliminary grasp of the general content, then conceptualize the main emotions contained in the design style, and finally determine the design style that matches the theme and content.
Positioning design theory plays an important role in book packaging design. Positioning design is reflected in modern book packaging design, which means that, in the book cover design and binding, the image and function of the book itself should be highlighted, and the fundamental 


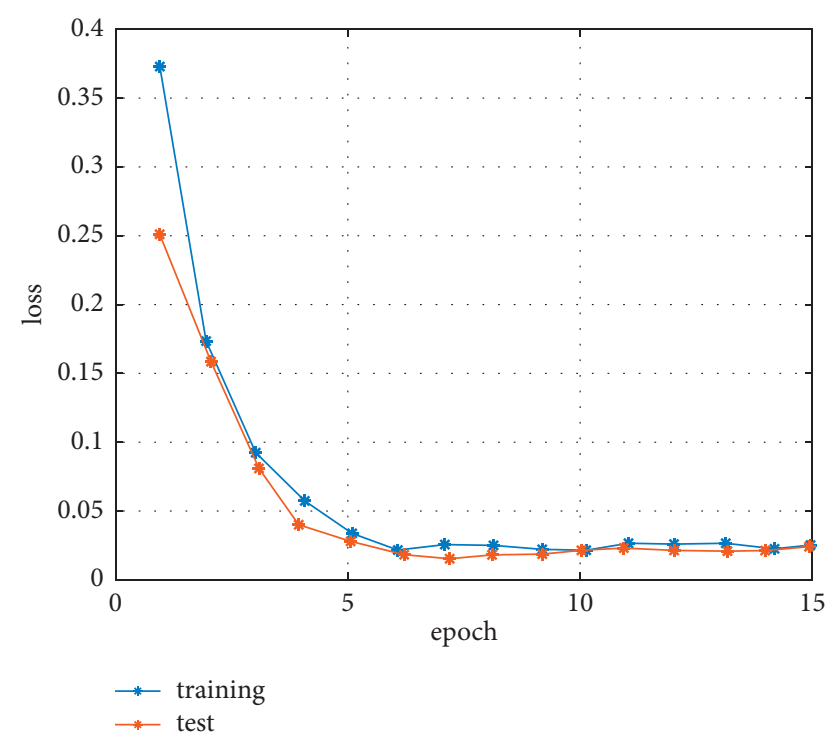

Figure 3: Convergence of loss function.

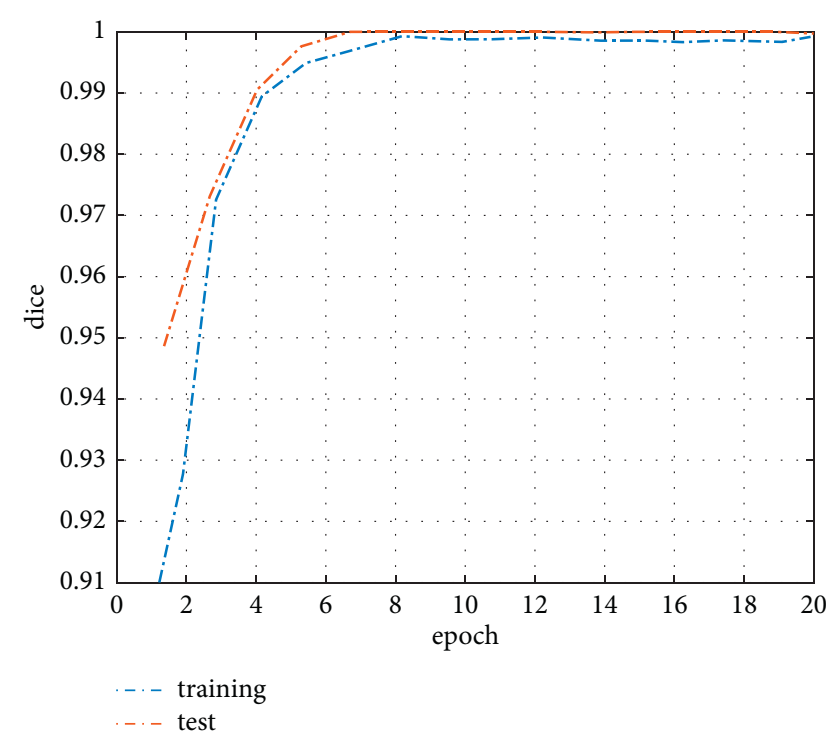

FIGURE 4: Dice curve.

function of the book should be emphasized, instead of focusing only on the image.

\section{Conclusions}

This paper describes the machine learning algorithms used in this paper, including the AdaBoost algorithm and SVR algorithm. Specifically, it includes the principles and specific implementation steps of the AdaBoost classification algorithm and SVR regression algorithm, as well as the evaluation metrics of AdaBoost classification and SVR regression analysis. Realization of physical books reflects artistry, creativity, professionalism, popularity, and the vitality of books in the packaging and design of books in the electronic information era. The stimulation effect of this paper starts from packaging design, inspection mechanism, and brand psychology to get the superior design in modern book packaging design.

\section{Data Availability}

The dataset used in this paper is available from the corresponding author upon request.

\section{Conflicts of Interest}

The authors declare that they have no conflicts of interest regarding this work.

\section{References}

[1] L. Han and Q. Tao, "Research on HPM teaching supported by hawgent dynamic mathematics software: take "the recognition of circle" as an example," Journal of Contemporary Educational Research, vol. 5, no. 8, pp. 148-154, 2021.

[2] N. Drew and P. Sternberge, By its Cover: Modern American Book Cover Design, Princeton Architectural Press, New York, NY, USA, 2005.

[3] T. Xie, C. Zhang, Z. Zhang, and K. Yang, "Utilizing active sensor nodes in smart environments for optimal communication coverage," IEEE Access, vol. 7, pp. 11338-11348, 2018.

[4] D. Çatal, "Usage of experimental typography of book cover design in digital age," New Trends and Issues Proceedings on Humanities and Social Sciences, vol. 2, no. 1, pp. 564-572, 2016.

[5] M. Manavi Rad and F. Morsali, "Relationship between economic capital and institutionalized cultural capital with the aesthetic taste of the graphics audiences relationship between economic capital and institutionalized cultural capital with the aesthetic taste of the graphics audiences case study: book cover design from the perspective of the audience in tehran," Painting Graphic Research, vol. 2, no. 2, pp. 15-28, 2019.

[6] M. Lachman, "Book cover inventory (in the latest literature)," Acta Universitatis Lodziensis. Folia Litteraria Polonica, vol. 44, no. 6, pp. 145-162, 2017.

[7] H. Jo, "The characteristics of magazine cover design in korea's early modern era," Archives of Design Research, vol. 27, no. 1, pp. 159-173, 2014.

[8] Z. Zhang, C. Zhang, M. Li, and T. Xie, "Target positioning based on particle centroid drift in large-scale WSNs," IEEE Access, vol. 8, pp. 127709-127719, 2020.

[9] J. M. Morgan-Brown, Localized Globality in Colonial Korean Literature: Ch'angjo as Part of the "Little Magazine" Movement, University of Toronto, Toronto, ON, Canada, 2020.

[10] K. Patton, Judging a Book by its Cover: Using Q Method to Examine Millennials' Perceptions and Expectations of Classic Novels, West Virginia University, Morgantown, WV, USA, 2012.

[11] X. Gong, B. Li, X. Chai, Y. Zhang, and M. Gu, "The application of dynamical management based on ontology-based simulation case-based description and reasoning," Communications in Computer and Information Science, vol. 324, pp. 386-394, 2012.

[12] T. Koichiro, U. Katsuya, I. Shuhei, and Y. Matsuo, "Model for evaluation of stock values by ensemble model using deep learning," Transactions of the Japanese Society for Artificial Intelligence, vol. 33, no. 1, pp. A-H51_1-11, 2018.

[13] O. Bhat and D. A. Khan, "Evaluation of deep learning model for human activity recognition," Evolving Systems, vol. 2021, Article ID 09373-6, 10 pages, 2021. 
[14] T. Chen and S. Lu, "Accurate and efficient traffic sign detection using discriminative adaboost and support vector regression," IEEE Transactions on Vehicular Technology, vol. 65, no. 6, pp. 4006-4015, 2015.

[15] Z. Zhuang, G. Liu, W. Ding et al., "Cardiac VFM visualization and analysis based on YOLO deep learning model and modified 2D continuity equation," Computerized Medical Imaging and Graphics, vol. 82, Article ID 101732, 2020.

[16] R.-J. Sun, M.-J. Fang, L. Tang et al., "CT-based deep learning radiomics analysis for evaluation of serosa invasion in advanced gastric cancer," European Journal of Radiology, vol. 132, Article ID 109277, 2020.

[17] B. Zhang, V. Velmayil, and V. Sivakumar, "A deep learning model for innovative evaluation of ideological and political learning," Progress in Artificial Intelligence, vol. 2021, Article ID 00253-3, 13 pages, 2021.

[18] W. Wei, C. Mcelroy, and S. Dey, "Using sensors and deep learning to enable on-demand balance evaluation for effective physical therapy," IEEE Access, vol. 99, p. 1, 2020.

[19] D. Gamdha, S. Unnikrishnakurup, K. J. J. Rose et al., “Automated defect recognition on X-ray radiographs of solid propellant using deep learning based on convolutional neural networks," Journal of Nondestructive Evaluation, vol. 40, no. 1, pp. 1-13, 2021.

[20] C. Zhang, T. Xie, K. Yang et al., "Positioning optimisation based on particle quality prediction in wireless sensor networks," IET Networks, vol. 8, no. 2, pp. 107-113, 2019.

[21] H. Li, D. Zeng, L. Chen, Q. Chen, M. Wang, and C. Zhang, "Immune multipath reliable transmission with fault tolerance in wireless sensor networks," in Proceedings of the International Conference on Bio-Inspired Computing: Theories and Applications, pp. 513-517, Springer, Beijing, China, November 2016.

[22] S. Mangaraj, T. K. Goswami, and P. V. Mahajan, “Applications of plastic films for modified atmosphere packaging of fruits and vegetables: a review," Food Engineering Reviews, vol. 1, no. 2, pp. 133-158, 2009.

[23] F. Shen, X. Zhao, G. Kou, and F. E. Alsaadi, "A new deep learning ensemble credit risk evaluation model with an improved synthetic minority oversampling technique," Applied Soft Computing, vol. 98, no. 1, Article ID 106852, 2021.

[24] W. Wang, M. Zhao, L. Wang, J. Huang, C. Cai, and X. Xu, "A multi-scene deep learning model for image aesthetic evaluation," Signal Processing: Image Communication, vol. 47, pp. 511-518, 2016.

[25] Y. Liu, Z. Zhang, X. Liu, L. Wang, and X. Xia, "Performance evaluation of a deep learning based wet coal image classification," Minerals Engineering, vol. 171, Article ID 107126, 2021.

[26] I. Hammad and K. El-Sankary, "Practical considerations for accuracy evaluation in sensor-based machine learning and deep learning," Sensors, vol. 19, no. 16, p. 3491, 2019.

[27] F. Geyer, "DeepComNet: performance evaluation of network topologies using graph-based deep learning," Performance Evaluation, vol. 130, pp. 1-16, 2019.

[28] C. Zhou, J. G. Chase, and G. W. Rodgers, "Degradation evaluation of lateral story stiffness using HLA-based deep learning networks," Advanced Engineering Informatics, vol. 39, pp. 259-268, 2019.

[29] Z. Wei, Z. Lu, Z. Wu, J. Wu, H. Zou, and S. Huang, “Toy-IoTOriented data-driven CDN performance evaluation model with deep learning," Journal of Systems Architecture, vol. 88, pp. 13-22, 2018.
[30] S. Li, "RETRACTED: deep adversarial model for musculoskeletal quality evaluation," Information Processing \& Management, vol. 57, no. 1, Article ID 102146, 2020.

[31] D. Wu, C. Zhang, L. Ji, R. Ran, H. Wu, and Y. Xu, "Forest fire recognition based on feature extraction from multi-view images," Traitement du Signal, vol. 38, no. 3, pp. 775-783, 2021.

[32] L. Wang, C. Zhang, Q. Chen et al., “A communication strategy of proactive nodes based on loop theorem in wireless sensor networks," in Proceedings of the 2018 Ninth International Conference on Intelligent Control and Information Processing (ICICIP), pp. 160-167, IEEE, Chongqing, China, November 2018.

[33] R. Žavbi and J. Rihtaršič, "Synthesis of elementary product concepts based on knowledge twisting," Research in Engineering Design, vol. 21, no. 2, pp. 69-85, 2010.

[34] J. Pavelka, "A book cover as an expression of conceptualization and a tool of social identity construction: the interpretation based on the example of G. Ritzer's book McDonaldization of society," World academy of science, engineering and technology, vol. 77, pp. 76-81, 2013. 